\title{
Exercise-Induced Anaphylaxis: An Update on Diagnosis and Treatment
}

\author{
Wojciech Barg • Wojciech Medrala • \\ Anna Wolanczyk-Medrala
}

Published online: 5 October 2010

(C) The Author(s) 2010. This article is published with open access at Springerlink.com

\begin{abstract}
Exercise-induced anaphylaxis (EIA) and fooddependent, exercise-induced anaphylaxis (FDEIA) are rare but potentially life-threatening clinical syndromes in which association with exercise is crucial. The range of triggering physical activities is broad, including as mild an effort as a stroll. EIA is not fully repeatable (ie, the same exercise may not always result in anaphylaxis in a given patient). In FDEIA, the combined ingestion of sensitizing food and exercise is necessary to precipitate symptoms. Clinical features and management do not differ significantly from other types of anaphylaxis. The pathophysiology of EIA and FDEIA is not fully understood. Different hypotheses concerning the possible influence of exercise on the development of anaphylactic symptoms are taken into consideration. These include increased gastrointestinal permeability, blood flow redistribution, and most likely increased osmolality. This article also describes current diagnostic and therapeutic possibilities, including changes in lifestyle and preventive properties of antiallergic drugs as well as acute treatment of these dangerous syndromes.
\end{abstract}

\footnotetext{
W. Barg

Department of Physiology, Medical University of Wroclaw, Wroclaw, Poland

W. Medrala $\cdot$ A. Wolanczyk-Medrala $(\square)$

Department of Internal Diseases, Geriatrics, and Allergology, Medical University of Wroclaw,

57 Traugutta Street,

50-417 Wroclaw, Poland

e-mail: wojmed@wp.pl

A. Wolanczyk-Medrala

Department of Clinical Research, Medical School of Legnica, Legnica, Poland
}

Keywords Exercise-induced anaphylaxis . Food-dependent, exercise-induced anaphylaxis . Basophil · Mast cell · Allergy · Food allergy · Histamine

\section{Introduction}

Anaphylaxis is defined as a potentially life-threatening generalized or systemic hypersensitivity reaction involving several organs and systems, particularly the skin, respiratory tract, gastrointestinal tract, and cardiovascular system [1]. It is believed that the first description of an anaphylactic reaction came from François Magendie in 1839 [2], while the term anaphylaxis was first used by Portier and Richet [3] in 1902.

There is no unified method of obtaining data about anaphylaxis; thus, its incidence is very difficult to evaluate clearly. Epidemiologic studies have reported a range of 8 to 50 per 100,000 person-years, with a lifetime prevalence of $0.05 \%$ to $2.0 \%$ [4]. A recent study from the United Kingdom reported the prevalence of anaphylaxis to be 32 in 100,000 [5]. General opinion suggests that the prevalence of anaphylaxis is underestimated and has increased in recent years. It is also presumed that there are about 50 to 2,000 episodes per 100,000 persons, and anaphylaxis might affect up to $2 \%$ of the Western population [6].

Since the early-1980s, interest has grown in patients with anaphylaxis triggered by exercise. The first case report came from Maulitz et al. [7] in 1979. They described a patient who experienced on two occasions anaphylactic symptoms caused by running that was preceded by shellfish ingestion 5 to $24 \mathrm{~h}$ earlier. Both strenuous exercise and causative food alone were well-tolerated. Kidd and coworkers [8] presented four such patients and designated the phenomenon of fooddependent, exercise-induced anaphylaxis (FDEIA). 
In 1980, Sheffer and Austen [9] presented a series of 16 patients in whom exertion elicited a variety of anaphylactic symptoms, including generalized urticaria, pruritus, angioedema, gastrointestinal colic, and hypotension. As this set of symptoms was very similar to anaphylactic syndrome resulting from contact with foreign antigen, they termed it exercise-induced anaphylaxis (EIA).

Patients with EIA are approximated to represent about $5 \%$ to $15 \%$ of all anaphylactic cases [10]. Prevalence of FDEIA is not well-documented, but it is estimated to make up one third to one half of all EIA patients. In a population of more than 76,000 Japanese junior high school students, Aihara et al. [11] found only $13(0.017 \%)$ and $24(0.031 \%)$ cases of FDEIA and EIA, respectively.

There is no known racial predilection for EIA. As for other cases of anaphylaxis, prevalence of EIA by gender changes with age. In the study by Aihara et al. [11], there was no gender predilection in EIA patients, while in the FDEIA group, the number of boys $(n=11)$ was significantly higher than that of girls $(n=2)$. When both children and adult EIA patients are considered, the overall incidence is highest in women. In two large studies of 199 and 279 EIA patients, respectively, the ratio of females to males was 2:1 [12,13]. Onset of EIA symptoms most typically occurs in young adulthood, predominantly in the second [12] or third [13] decade of life, but may vary from younger than 5 years of age to older than 75 years of age.

Symptoms of EIA are usually triggered by exercise of moderate intensity. Activities most commonly considered to be causative of EIA (ie, the most often reported to be associated with EIA) are listed in Table 1. There is no entirely safe exercise for patients with EIA. Symptoms may develop just as commonly in individuals performing mild physical effort such as yard work as they do in vigorously exercising athletes. Exercise with less cardiovascular demand seems to be safer and is responsible for less than 2\% of EIA episodes [13]. Episodes of EIA are not fully predictable. In some patients, exercise of the same intensity sometimes provokes symptoms, but on other occasions, a patient will remain symptom free. It seems plausible that some external factors may influence EIA. Warm environment, high humidity, and cold environment have been reported to be associated with EIA occurrence among 64\%,
$32 \%$, and $23 \%$ of patients, respectively [12]. Frequency of EIA events varies from patient to patient and ranges from singular episodes to multiple episodes too frequent to enumerate. Shadick et al. [13] reported an average of 14.5 attacks per year. Most patients claim that the frequency of EIA episodes remains stable or decreases after the illness first begins. The most frequently reported symptoms are listed in Table 2.

In the subpopulation of patients with FDEIA, ingestion of causative food and physical effort are necessary to induce anaphylaxis. In Europeans, tomatoes, cereals, and peanuts are the most frequent allergenic foods [14], whereas in the Japanese population, wheat and particularly the omega- 5 gliadin allergen are the most frequent $[15,16 \bullet]$. Other causative foods include seafood (especially shellfish), seeds, cow's milk, some vegetables and fruits (eg, oranges, onions, or grapes), foods contaminated with aeroallergens such as house dust mite and Penicillium mold, meats, and miscellaneous foods (eg, alcohol, snails, taro, red bean, and mushrooms) [10].

Symptoms of EIA may start at any stage of exercise or after it, but in $90 \%$ patients, they begin within $30 \mathrm{~min}$ after initiating exercise [13]. In FDEIA, ingestion of causative food usually precedes exercise by several minutes or even hours. However, some observations indicate that FDEIA may also occur if the food is ingested soon after the completion of exercise. Thus, it seems very likely that in FDEIA, not the sequence but rather the coincidence of triggering factors is of crucial importance.

Clinical history of EIA does not differ significantly from that of anaphylaxis triggered by other factors. In the historic paper by Sheffer and Austen [9], four stages of an EIA event were distinguished: prodromal, early, fully developed, and late. The prodromal symptoms manifest with fatigue and prostration and generalized pruritus with erythema. The early stage is characterized by generalized urticaria. If the event progresses, fully developed EIA includes gastrointestinal symptoms with abdominal cramps, nausea, and vomiting, as well as symptoms of upper airway obstruction with dyspnea, stridor, and a feeling of choking. Symptoms of the late phasefrontal headache and fatigue - may be present up to $72 \mathrm{~h}$ after the onset of EIA. Of course, this description does not include all manifestations. In a fully developed EIA attack, the spectrum of symptoms is wider and may be much more severe. Some
Table 1 Activities associated with symptoms of exerciseinduced anaphylaxis

\begin{tabular}{lll}
\hline Activity & Wade et al. [12], $n(\%)$ & Shadick et al. [13], $n(\%)$ \\
\hline Jogging & $138(69)$ & $219(78)$ \\
Aerobics & $70(35)$ & - \\
Walking & $59(30)$ & $117(42)$ \\
Tennis/racquetball & - & $78(28)$ \\
Tennis & $41(21)$ & - \\
Racquetball & $31(16)$ & - \\
Dancing & $48(24)$ & $73(26)$ \\
Bicycling & $37(19)$ & $68(24)$ \\
\hline
\end{tabular}


Table 2 Frequency of exerciseinduced anaphylaxis symptoms

\begin{tabular}{lll}
\hline Symptom & Wade et al. [12], $n(\%)$ & Shadick et al. [13], $n(\%)$ \\
\hline Pruritus & $183(92)$ & $257(92)$ \\
Urticaria & $166(83)$ & $241(86)$ \\
Angioedema & $157(78)$ & $201(72)$ \\
Flushing & $150(75)$ & $194(70)$ \\
Shortness of breath & $117(59)$ & $141(51)$ \\
Dysphagia & - & $94(34)$ \\
Chest tightness & - & $92(33)$ \\
Loss of consciousness & $64(32)$ & $90(32)$ \\
Diaphoresis & $86(43)$ & $90(32)$ \\
Headache & $59(30)$ & $78(28)$ \\
Nausea/diarrhea/colic & $59(30)$ & $77(28)$ \\
Choking/throat constriction/hoarseness & - & $71(25)$
\end{tabular}

patients develop symptoms from the lower airways, including dyspnea, wheezing, and chest tightness. Cardiovascular symptoms, including collapse or altered consciousness, are reported in one third of EIA patients [13]. Fatalities or nearfatalities are very rare [17-19], but EIA must be considered as a potentially life-threatening condition.

\section{Diagnosis}

A diagnosis of EIA is made based on clinical history and physical examination. Patients are diagnosed with EIA if they have had anaphylactic symptoms associated with exercise: hives and/or angioedema or cardiovascular collapse, with or without other anaphylactic symptoms such as gastrointestinal disorders and upper or lower airway obstruction. If an association of anaphylactic symptoms with exercise is proven, it must be clearly stated whether it is food-dependent (FDEIA) or food-independent EIA. This is of crucial importance to the prevention of future EIA attacks. The clinical history must be carefully acquired to distinguish episodes of EIA from exercise-accompanied anaphylaxis. In some individuals, symptoms of anaphylaxis may repeat during exercise due to factors other than physical effort. Examples include exposure to cold while swimming, ingestion of NSAIDs as painkillers by vigorously exercising athletes, and exposure to sensitizing contact allergen (eg, latex). If the symptoms are not obvious, a differential diagnosis should be considered. A clinician should take into account not only other types of anaphylaxis but also disorders that can mimic anaphylactic symptoms. Differential diagnosis should include disorders listed in the following sections.

Idiopathic Anaphylaxis

The trigger of idiopathic anaphylaxis is unknown and unpredictable, but the symptoms are entirely the same as those observed in EIA. Because low-intensity exertion may provoke EIA, a very careful history taking is of crucial importance for distinguishing between those two forms of anaphylaxis.

\section{Cholinergic Urticaria}

Cholinergic urticaria results from increasing body temperature, either active (eg, exercise) or passive (eg, hot shower). Lesions are restricted to skin and manifested as punctuate, pinpointsized wheals, whereas in EIA, they are usually much larger and diffused. Bronchoconstriction with wheezing may occur, but neither angioedema nor hypotension is present.

\section{Cold Urticaria}

Skin lesions are similar to those in EIA. They start in places exposed to cold, usually on the hands, face, and neck, and promptly take up the whole body. Intensive exposure to cold (eg, contact with cold water) may induce a robust histamine release, resulting in cardiovascular symptoms with lowered blood pressure, collapse, or even shock. Cold urticaria can result from exercise with exposure to cold (eg, swimming or outdoor activity in winter).

\section{Mastocytosis}

Mastocytosis is a rare and heterogenic disorder characterized by uncontrolled proliferation and accumulation of mast cells in the skin (cutaneous mastocytosis) or more organs (generalized mastocytosis). Skin involvement is manifested with urticaria pigmentosa, whereas in generalized mastocytosis, gastrointestinal symptoms with cramps, bloating, and sometimes diarrhea, hypotension, and psychiatric disorders may be present. These symptoms are associated with mediator release; thus, the risk of severe anaphylactic reactions is increased, though they are not clearly provoked by exertion. The diagnosis is made based on 
the elevated serum $\alpha$-tryptase and presence of mast cell hyperplasia.

\section{Hereditary Angioedema}

Hereditary angioedema is a rare autosomal dominant illness associated with $\mathrm{C}_{1}$-inhibitor protein disorder, either decreased serum level or presence of abnormal protein. The symptoms may be triggered by major exertion, but in contrast to EIA, the edematic lesions are nonpitting and produce no itching.

\section{Exercise-Induced Asthma}

Exercise-induced asthma is manifested with typical symptoms of asthma exacerbation resulting from exercise. Consequently, the symptoms are limited to the lower airways, except in lifethreatening asthma. In contrast to EIA, exercise-induced asthma is fully preventable with inhaled, rapid-acting $\beta$-agonists prior to exercise or regularly inhaled glucocorticosteroids.

\section{Neoplasmatic Disorders}

In some neoplasmatic disorders with endocrine activity, such as carcinoid, phaeochromocytoma, medullary carcinoma of the thyroid and pancreatic cell tumor, release of bioactive substances may mimic anaphylactic reactions. Although they are not exercise dependent, the release may be triggered by exertion. Skin manifestations with generalized rush or pallor are very rapid, and cardiovascular symptoms are predominant.

\section{Management}

\section{Prophylaxis}

Avoidance of potentially precipitating factors is of crucial importance. Thus, patients should not perform outdoor exercises during very cold, hot, or humid weather, or, in those with seasonal atopy, during the pollen season. Aspirin and other NSAIDs should not be taken in association with exercise. Refraining from exercise for 4 to $6 \mathrm{~h}$ after food ingestion is a classical recommendation for those with FDEIA. Some evidence suggests that in FDEIA, the sequence of events might be reversed (ie, anaphylaxis may occur after ingestion of causative food following prolonged exercise). Thus, it seems reasonable that patients with FDEIA should refrain not only from postprandial exertion but from food ingestion after exercising as well.

Some pharmacologic protection also seems possible. A case report demonstrated a protective effect of coadministration of cetirizine and montelukast in an adolescent male with FDEIA triggered by peaches [20]. Recently, evidence has started to accumulate indicating that pretreatment with agents that can inhibit cell degranulation may have a preventive effect in FDEIA. It has been demonstrated that in FDEIA to wheat, administration of sodium cromoglycate before ingestion of the causative food and exercise prevented symptoms of anaphylaxis in two children [21•]. A similar effect was observed in a young female after pretreatment with ketotifen [22].

\section{Acute Treatment}

Management of EIA does not differ from that of other types of anaphylaxis [23]. Immediate termination of physical effort at the earliest warning manifestation is of crucial importance to avoid potentially life-threatening cardiovascular symptoms. Base pharmacotherapy includes epinephrine, antihistamines, and systemic corticosteroids. Shadick et al. [13] reported use of those drugs by $31 \%, 56 \%$, and (surprisingly) only $5 \%$ of patients with EIA, respectively.

\section{Pathophysiology}

The pathophysiology of EIA and FDEIA is not fully understood. There is general agreement that histamine release is the key feature. Increased plasma histamine has been documented during EIA $[24,25]$ and FDEIA [11] episodes. Following exercise, cutaneous mast cells from EIA patients - but not from controls - presented morphologic changes similar to those observed in atopic patients after allergen stimulation [26]. What is the exercise-specific factor (or, possibly, combination of factors) responsible for cell degranulation that is not well-understood? Current working hypotheses include those outlined below.

\section{Increased Gastrointestinal Permeability}

Exercise increases absorption from the gastrointestinal tract. Although the importance of altered intestinal permeability is still debatable [27], it is speculated that allergens have enhanced contact with the gut-associated immune system. In some cases, FDEIA symptoms depend on the amount of causative food ingested [28]. Increased permeability might also result in absorption of only partially digested allergenic proteins. It has been demonstrated that gliadin, the predominant allergen in wheat-dependant EIA, appears in sera from FDEIA patients and healthy controls after an exercise/food challenge, but not after food ingestion alone [29].

Aspirin and NSAID Ingestion

NSAIDs have been shown in aspirin models to induce FDEIA symptoms with co-ingestion of the causative food [30]. Two mechanisms should be taken into consideration. First, it was 
proven that aspirin increases gastrointestinal permeability and antigen uptake [29], even in a dose as low as $100 \mathrm{mg}$ [31]. Second, aspirin itself might enhance immune cell degranulation. Skin prick test amelioration due to oral aspirin intake was observed in five of eight FDEIA patients [32], possibly due to cyclooxygenase inhibition. Of course, aspirin intake definitely is not a universal factor responsible for anaphylactic symptoms, but it may provide interesting insights into the pathomechanisms of FDEIA and may be used as a substitute for exercise in an experimental model.

\section{Increased Tissue Enzyme Activity}

Tissue transglutaminase in the intestinal mucosa could be activated by exercise and aspirin. Omega- 5 gliadin, a major allergen in FDEIA to wheat, is cross-linked by tissue transglutaminase, which results in the formation of large peptide aggregates and facilitates greater IgE cross-linking [33]. It seems likely that this could elicit allergic reactions in patients with FDEIA to wheat; however, no experiments have confirmed the presence of gliadin-transglutaminase complexes in the circulating blood.

\section{Blood Flow Redistribution}

It is well-known that even in mild exercise, a redistribution of blood flow occurs from inactive to active tissues. Cooper and coworkers [34] suggested that food-sensitized, gutassociated immune cells do not elicit symptoms of anaphylaxis as long as they remain in a local (portal) circulation. However, when these sensitized cells are shifted to the skin and skeletal muscle with exercise-induced blood flow redistribution, FDEIA symptoms are present. This hypothesis was recently developed by Robson-Ansley and Toit [35•], who speculated that absorbed food allergens are well-tolerated by intestinal mast cells and thus provoke no symptoms at rest. The exercise-induced redistribution of blood results in displacement of the recently ingested allergens from the gut to the target tissues. Mast cells in the skin and skeletal muscles are phenotypically different from those in the gut [36] and can be induced by the food allergens, increasing the potential for EIA. An argument against this hypothesis is a protective action of sodium cromoglycate against the precipitation of clinical symptoms of FDEIA [21•]. Because this drug inhibits activation of intestinal mast cells, but not skin mast cells [37, 38], it clearly indicates the leading role for intestinal mast cells in initiation of a further cascade of pathological symptoms.

\section{Increased Osmolality}

The first step in the cascade of FDEIA events most likely depends on intestinal mast cell activation caused by increased osmolality of the microenvironment. It is known that intestinal blood flow, if increased by administration of vasodilators, significantly decreases tissue osmolality [39]. Tissue osmolality measured at the villous tips was 1,000 to $1,200 \mathrm{mOsm} / \mathrm{kg} \mathrm{H} \mathrm{H}_{2} \mathrm{O}$, while at the villous bases, it was approximately isotonic to plasma. In intestinal blood vessels, exercise-induced blood flow redistribution may subsequently increase tissue osmolality at the villous bases and consequently activate mast cells. In our earlier experiment, we demonstrated that in basophils from FDEIA patients, increased osmolality results in increased histamine release compared with atopic patients and healthy volunteers [40••]. In physiologic osmolality of $280 \mathrm{mOsm}$, basophil activation determined as CD203c expression on their surface does not differ among FDEIA patients, atopic patients, and healthy controls. In contrast, osmolality increased to 340 mOsm resulted in greater than twofold higher basophil activation in FDEIA patients compared with the other individuals investigated [41•]. Secretion of histamine and other vasoactive mediators results in allergen absorption and its distribution to other organs, such as skin, which leads to augmented IgE-dependent activation of mast cells and spreads the symptoms of anaphylaxis. This proposal is in accordance with previous observations concerning the influence of aspirin on food allergen absorption and skin mast cell reactivity [32] as well as with an earlier observation concerning increased skin mast cell reactivity to codeine after physical exercise seen only in FDEIA syndrome [42].

Whatever the mechanism(s) is, exercise increases gut permeability, resulting in an increased amount of food allergen presented to the mast cells and basophils. However, there is still an open question as to why in FDEIA patients the cells are activated and subsequently produce severe anaphylactic reactions, while in patients with food urticaria or fooddependent atopic dermatitis, they remain silent and do not influence the intensity of symptoms. Therefore, it is likely that the immune systems of FDEIA patients have a "unique property" that elicits different responses to the stimuli. On the basis of our results $[40 \bullet \bullet$, we hypothesize that this "unique property" might be an individually decreased threshold for histamine release. In addition, there are two other hypotheses that are not well-documented and seem to be of minor importance.

\section{Increased Endogenous Endorphin Release}

Endogenous endorphins are known to enhance mast cell degranulation [43], but a significant increase in serum endorphins is observed with prolonged and strenuous exercise [44]. Thus, it seems unlikely that within the short effort of mild intensity that is sufficient to initiate EIA, serum endorphins might rise up enough to trigger cell degranulation. 


\section{Alterations in Plasma $\mathrm{pH}$}

In contrast to prolonged and strenuous exercise, exertion of moderate intensity does not alter blood $\mathrm{pH}$ significantly [45]. Only two cases have reported inhibition of FDEIA symptoms with sodium bicarbonate $[46,47]$. The mechanism in which increased serum $\mathrm{pH}$ prevents anaphylaxis remains unclear and requires further research.

\section{Conclusions}

Pathomechanism, or more likely pathomechanisms, of EIA and FDEIA remain incompletely understood. Data from the recent investigations indicate that the cascade of events resulting in FDEIA symptoms is triggered in the intestine. Various activating factors are taken into consideration, but exerciseinduced, locally increased osmolality in the intestine seems the most probable. In patients with FDEIA, hyperosmolality enhances mast cell degranulation and thus increases gut permeability, resulting in an increased amount of food allergen(s) presented to the mast cells and basophils.

There is still an open question as to why these cells are activated in FDEIA, but not in other pathologies associated with food allergy. In patients with FDEIA, higher than normal susceptibility to activation caused by even small changes resulting from exercise in the microenvironment seems the most likely supposition.

The proposed FDEIA pathophysiology, despite being suggestive and in agreement with other accumulated data, requires prospective studies to increase the number of observations; however, the rarity of this syndrome has not facilitated these efforts.

Disclosure No potential conflicts of interest relevant to this article were reported.

Open Access This article is distributed under the terms of the Creative Commons Attribution Noncommercial License which permits any noncommercial use, distribution, and reproduction in any medium, provided the original author(s) and source are credited.

\section{References}

Papers of particular interest, published recently, have been highlighted as:

- Of importance,

-• Of major importance

1. Johansson SG, Hourihane JO, Bousquet J, et al.: A revised nomenclature for allergy. Allergy 2001, 56:813-824.
2. Saavedra-Delgado AM: François Magendie on anaphylaxis (1839). Allergy Proc 1991, 12:355-356.

3. Portier P, Richet C: De l'action anaphylactique de certains venins [in French]. C R Séances Soc Biol 1902, 54:170.

4. Tang ML, Osborne N, Allen K: Epidemiology of anaphylaxis. Curr Opin Allergy Clin Immunol 2009, 9:351-356.

5. Capps JA, Sharma V, Arkwright PD: Prevalence, outcome and pre-hospital management of anaphylaxis by first aiders and paramedical ambulance staff in Manchester, UK. Resuscitation 2010, 81:653-657.

6. Lieberman P: Epidemiology of anaphylaxis. Curr Opin Allergy Clin Immunol 2008, 8:316-320.

7. Maulitz RM, Pratt DS, Schocket AL: Exercise-induced anaphylactic reaction to shellfish. J Allergy Clin Immunol 1979, 63:433-434.

8. Kidd III JM, Cohen SH, Sosman AJ, et al.: Food-dependent exercise-induced anaphylaxis. J Allergy Clin Immunol 1983, 71:407-411.

9. Sheffer AL, Austen KF: Exercise-induced anaphylaxis. J Allergy Clin Immunol 1980, 6:106-111.

10. Du Toit G: Food-dependent exercise-induced anaphylaxis in childhood. Pediatr Allergy Immunol 2007, 18:455-463.

11. Aihara Y, Takahashi Y, Kotoyori T, et al.: Frequency of fooddependent, exercise induced anaphylaxis in Japanese junior-highschool students. J Allergy Clin Immunol 2001, 108:1035-1039.

12. Wade JP, Liang MH, Sheffer AL: Exercise-induced anaphylaxis: epidemiologic observations. Prog Clin Biol Res 1989, 297:175182.

13. Shadick NA, Liang MH, Partridge AJ, et al.: The natural history of exercise-induced anaphylaxis: survey results from a 10-year follow-up study. J Allergy Clin Immunol 1999, 104:123-127.

14. Romano A, Di Fonso M, Giuffreda F, et al.: Food-dependent exercise-induced anaphylaxis: clinical and laboratory findings in 54 subjects. Int Arch Allergy Immunol 2001, 125:264-272.

15. Morita E, Kunie K, Matsuo H: Food-dependent exercise-induced anaphylaxis. J Dermatol Sci 2007, 47:109-117.

16. • Morita E, Matsuo H, Chinuki Y, et al.: Food-dependent exerciseinduced anaphylaxis - importance of omega-5 gliadin and HMWglutenin as causative antigens for wheat-dependent exerciseinduced anaphylaxis. Allergol Int 2009, 58:493-498. This recent and comprehensive study demonstrated the molecular basis of wheat-dependent EIA.

17. Noma T, Yoshizawa I, Ogawa N, et al.: Fatal buckwheat dependent exercised-induced anaphylaxis. Asian Pac J Allergy Immunol 2001, 19:283-286.

18. Flannagan LM, Wolf BC: Sudden death associated with food and exercise. J Forensic Sci 2004, 49:543-545.

19. Attenhofer C, Speich R, Salomon F, et al.: Ventricular fibrillation in a patient with exercise-induced anaphylaxis, normal coronary arteries, and a positive ergonovine test. Chest 1994, 105:620-622.

20. Peroni DG, Piacentini GL, Piazza M, et al.: Combined cetirizinemontelukast preventive treatment for food-dependent exercise-induced anaphylaxis. Ann Allergy Asthma Immunol 2010, 104:272-273.

21. - Sugimura T, Tananari Y, Ozaki Y, et al.: Effect of oral sodium cromoglycate in 2 children with food-dependent exercise-induced anaphylaxis (FDEIA). Clin Pediatr (Phila) 2009, 48:945-950. This was a case report demonstrating the prevention of FDEIA symptoms with intestinal mast cell inhibition.

22. Choi JH, Lee HB, Ahn IS, et al.: Wheat-dependent, exerciseinduced anaphylaxis: a successful case of prevention with ketotifen. Ann Dermatol 2009, 21:203-205.

23. Lieberman P, Decker W, Camargo CA Jr, et al.: SAFE: a multidisciplinary approach to anaphylaxis education in the emergency department. Ann Allergy Asthma Immunol 2007, 98:519-523.

24. Lewis J, Lieberman P, Treadwell G, et al.: Exercise-induced urticaria, angioedema, and anaphylactoid episodes. J Allergy Clin Immunol 1981, 68:432-437. 
25. Sheffer AL, Soter NA, McFadden ER Jr, et al.: Exercise-induced anaphylaxis: a distinct form of physical allergy. J Allergy Clin Immunol 1983, 71:311-316.

26. Sheffer AL, Tong AKF, Murphy GF, et al.: Exercise-induced anaphylaxis: a serious form of physical allergy associated with mast cell degranulation. J Allergy Clin Immunol 1985, 75:479-484.

27. Ménard S, Cerf-Bensussan N, Heyman M: Multiple facets of intestinal permeability and epithelial handling of dietary antigens. Mucosal Immunol 2010, 3:247-259.

28. Hanakawa Y, Tohyama M, Shirakata Y, et al.: Food-dependent exercise-induced anaphylaxis: a case related to the amount of food allergen ingested. Br J Dermatol 1998, 138:898-900.

29. Matsuo H, Morimoto K, Akaki T, et al.: Exercise and aspirin increase levels of circulating gliadin peptides in patients with wheat-dependent exercise-induced anaphylaxis. Clin Exp Allergy 2005, 35:461-466.

30. Harada S, Horikawa T, Ashida M, et al.: Aspirin enhances the induction of type I allergic symptoms when combined with food and exercise in patients with food dependent exercise-induced anaphylaxis. Br J Dermatol 2001, 145:336-339.

31. Matsuo H, Kaneko S, Tsujino Y, et al.: Effects of nonsteroidal anti-inflammatory drugs (NSAIDs) on serum allergen levels after wheat ingestion. J Dermatol Sci 2009, 53:241-243.

32. Aihara M, Miyazawa M, Osuna $H$, et al.: Food-dependent exercise-induced anaphylaxis: influence of concurrent aspirin administration on skin testing and provocation. Br J Dermatol 2002, 146:466-472.

33. Palosuo K, Varjonen E, Nurkkala J, et al.: Transglutaminasemediated cross-linking of a peptic fraction of omega-5 gliadin enhances IgE reactivity in wheat-dependent, exercise-induced anaphylaxis. J Allergy Clin Immunol 2003, 111:1386-1392.

34. Cooper DM, Radom-Aizik S, Schwindt C, et al.: Dangerous exercise: lessons learned from dysregulated inflammatory responses to physical activity. J Appl Physiol 2007, 103:700-709.

35. - Robson-Ansley P, Toit GD: Pathophysiology, diagnosis and management of exercise-induced anaphylaxis. Curr Opin Allergy Clin Immunol 2010, 10:312-317. This was a recent and comprehensive review of EIA.

36. Welle M: Development, significance, and heterogeneity of mast cells with particular regard to the mast cell-specific proteases chymase and tryptase. J Leukoc Biol 1997, 61:233-245.
37. Okayama Y, Church MK: Comparison of the modulatory effect of ketotifen, sodium cromoglycate, procaterol and salbutamol in human skin, lung and tonsil mast cells. Int Arch Allergy Immunol 1992, 97:216-225.

38. Okayama Y, Benyon RC, Rees PH, et al.: Inhibition profiles of sodium cromoglycate and nedocromil sodium on mediator release from mast cells of human skin, lung, tonsil, adenoid and intestine. Clin Exp Allergy 1992, 22:401-409.

39. Jodal M, Hallbäck DA, Lundgren O: Tissue osmolality in intestinal villi during luminal perfusion with isotonic electrolyte solutions. Acta Physiol Scand 1978, 102:94-107.

40. •- Barg W, Wolanczyk-Medrala A, Obojski A, et al.: Fooddependent exercise-induced anaphylaxis: possible impact of increased basophil histamine releasability in hyperosmolar conditions. J Investig Allergol Clin Immunol 2008, 18:312-315. This was the first demonstration of decreased threshold for histamine release from basophils in an FDEIA patient.

41. - Wolanczyk-Medrala A, Barg W, Gogolewski G, et al.: Influence of hyperosmotic conditions on basophil CD203c upregulation in patients with food dependent exercise-induced anaphylaxis. Ann Agric Environ Med 2009, 16:301-304. This study demonstrated the influence of increased osmolality on basophil activation.

42. Lin RY, Barnard M: Skin testing with food, codeine, and histamine in exercise-induced anaphylaxis. Ann Allergy 1993, $70: 475-478$.

43. Casale TB, Bowman S, Kaliner M: Induction of human cutaneous mast cell degranulation by opiates and endogenous opioid peptides: evidence for opiate and nonopiate receptor participation. J Allergy Clin Immunol 1984, 73:775-781.

44. Schwarz L, Kindermann W: Changes in beta-endorphin levels in response to aerobic and anaerobic exercise. Sports Med 1992, $13: 25-36$.

45. Del Coso J, Hamouti N, Aguado-Jimenez R, et al.: Restoration of blood $\mathrm{pH}$ between repeated bouts of high-intensity exercise: effects of various active-recovery protocols. Eur J Appl Physiol 2010, 108:523-532.

46. Azofra GJ, Sastre DJ, Olaguibel Rivera JM, et al.: Exerciseinduced anaphylaxis: inhibition with sodium bicarbonate. Allergy 1986, 41:471.

47. Katsunuma T, Iikura Y, Akasawa A, et al.: Wheat-dependent exercise-induced anaphylaxis: inhibition by sodium bicarbonate. Ann Allergy 1992, 68:184-188. 